\title{
Design and Fabrication of MEMS Humidity Sensor using Graphene Oxide
}

\author{
L. Chitra
}

\begin{abstract}
This work describes the consequence of graphene oxide on the paddle shaped cantilever structure for the measurement of humidity in oil condition monitoring .The oxides present in the graphene has an significant effect in absorbing the content of water that available in the earth. The model for humidity sensor is designed and simulated using comsol Multiphysics. Various structures were simulated and the best structure is chosen for designing the humidity sensor. Graphene oxide was chemically synthesized from graphite powder. Thin film of $G O$ was deposited on Aluminium substrate using spin coating. Various materials were analysed and the best material is coated on the substrate for humidity sensing. The samples were characterized using Scanning electron Microscope and Xray Diffraction methods. The sensitivity is ten times better than the conventional humidity sensor. The response time is fast less than $1 / 4$ of the conventional type. The recovery time of the graphene oxide based humidity sensor is also less than $1 / 2$ of the conventional one.
\end{abstract}

Keywords: Comsol Multiphysics, Cantilever, Comb drive capacitor, Diaphragm, Membrane, MEMS, thermal actuator, Stress.

\section{INTRODUCTION}

M onitoring the parameters of lubrication oil present in the gear box lubricating system of the windmill is an important task. The parameter such as wear particles, humidity and presence of $\mathrm{O} 2$ in the oil should be monitored continuously so that the reliability of the wind farm is maintained. These parameters only ensure the health of the gearbox. Also, after a long period of time, the nature of the oil changes due to changes in viscosity. The continuous assessment should be done on the oil using various sensors to monitor viscosity, humidity and presence of wear particles. The innovation and development of the micro technology provides the opportunity to design a micro sensor for measuring the humidity and it can be easily fabricated with the electronic components which enable the data to transfer from inside the gear box lubricating system to the monitoring station.

In porous materials, the surface and pores present in the layer absorb the moisture through some physical or chemical reactions. The properties of the material also depend on the size of the pore and grains present in the layer.

The conductivity of the pores present in the layer changes

Revised Manuscript Received on December 30, 2019.

Dr. L. Chitra *, Associate Professor, Department of Electrical and Electronics Engineering Aarupadai Veedu Institute of Technology, Chennai, India. even for a small change in humidity. The requirement of humidity sensor is it should have a wide sensitivity throughout the range, fast response for a small change in humidity, accuracy in repeated measurements, mechanical strength should be good, no reaction to impurities present in the atmosphere, small structure and low cost.

There are many classifications which satisfies the above requirements. They are organic, inorganic materials, polymers, electrolytes and ceramics. Each type has its own advantages and disadvantages. The sensitivity of the sensor built upon the surface area in which the micro-pores are distributed. The sensitivity can be adjusted by properly selecting the dimensions of the particle present in the starting material. Also it depends on the other process such as forming and sintering. The porous structure is having grains and pores formed during the process of sintering. The pores present in the surface are important in measuring the humidity since the different radii of the pores is a function of relative humidity. The condensation of the water molecules takes place inside the pores of different radii.

The presence of functional groups such as carboxyl, carbonyl and hydroxyl makes it hydrophilic. The hydrophilic nature makes it completely soluble in water. So it is used for humidity sensing. In this paper, humidity sensor is designed in paddle shape, with a coating of grapheme oxide with two leads of wire. Due to the absorption of water molecules on the surface of the grapheme oxide, the impedance of the layer changes. As the humidity changes, the resistance between the leads also changes. So this can acts as humidity sensor.

The common materials used in the design of humidity sensor are polymers, metal oxides which absorb moisture

Metal oxide humidity sensors have superior advantages when compared to polymer type of humidity sensors. The important advantages are its potential power, hot capability and protection to chemicals. The ceramic materials also divided into macro porous, meso-porous and micro-porous. The meso-porous is considered to be the best size for the absorption of humidity which is having the size in the range of 2 to $50 \mathrm{~nm}$.Porosity is an important parameter since it decides the active portions for the absorption of moisture. The time taken for the response of the sensor also depends on the porosity [1].

Graphene oxide film is also used for humidity sensing Compared to the performance of grapheme oxide and metal oxides it is found that go type has good sensitivity [2].

\section{PROPOSED METHODOLOGY}

\section{A. Sensor Design}

The article presented a MEMS

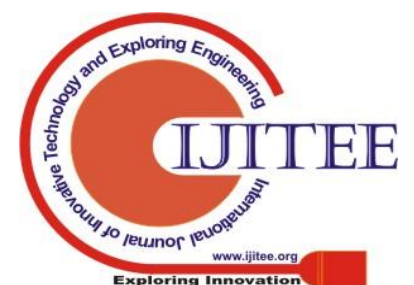


humidity sensor for online monitoring of moisture gratification of substance in the grtease (oil). The deformation of the sensor is analysed and simulated. When there is a presence of moisture, the mass of the cantilever increases and hence there is a deflection. The sensitivity of the structure and sensor output can be analysed [3-5].

Micro-cantilever sensors are the simplest and most promising structure of design used in the measurement of humidity. The basic principle of humidity sensor is measuring the deformation or bending of the cantilever beam. The mass of the cantilever increases when the absorption of analytes gets increased. The increased mass of the cantilever is due to the binding of analytes present. Thus qualitative and quantitative analysis can be carried out [7-10].

MEMS Humidity sensor is designed with a thin sensing film over the substrate. The film is made up of graphene oxide. Graphene oxide is set by customized hummer's technique [19-20]. The paddle shaped cantilever structure is designed and the graphene oxide film is formed by spin coating. The absorption of humidity by the thin film coated on the substrate of the sensor increases the bending of the beam. This film absorbs the water molecules. The change in the amount of water vapour molecules present in the oil of lubricating system makes some physical and chemical interactions with the sensing film and there is a change in deformation due to the absorption or diffusion process [11-14].

The sensor should be fabricated such a way the sensitivity has a good linear response. The deflection and maximum deflection or deformation can be designed hypothetically by the method as shown in equation (1) and (2).The material used for the fabrication of the sensing film was graphene oxide.

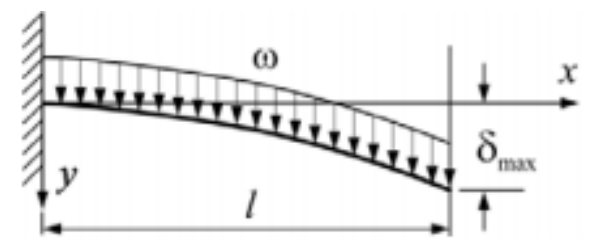

Fig. 1.Deformation of the MEMs cantilever structure

Deflection at any section

$$
Y=\frac{\omega x^{2}}{24 E I}\left(x^{2}+6 l^{2}-4 l x\right)
$$

Max deflection

$$
\delta_{\max }-\frac{\omega l^{4}}{8 E I}
$$

\footnotetext{
$\mathrm{Y}=$ deflection

$\omega=$ distributed load

$\mathrm{E}=$ modulus of elasticity

$\mathrm{i}=$ length of the cantilever beam

$\mathrm{I}=$ moment of Inertia

$\mathrm{E}=$ modulus of elasticity
}

\section{B. Sensor Design Optimization}

This paper explains the simulated model of cantilever type humidity sensor. The cantilever sensor is made up of polypropylene substrate. The prepared graphene oxide is coated over the substrate acts 9:02 AMas the sensing material []15-16]. The water vapour present in the oil is absorbed by the graphene oxide and there is an expansion in the structure. The expansion forces the cantilever beam to bend and induces stress .The stress induced because of graphene oxide swelling is proportional to the relative humidity. The model described is simulated using Comsol Multiphysics [17-18].

First analyses were done for designing the structure of the sensor. Various structures such as rectangular type, V shape and Paddle type MEMS humidity sensor were designed and simulated using Comsol Multiphysics. It is proven that the paddle shaped structure has good sensitivity towads the deformation compared to rectangular and $\mathrm{v}$ shaped structure [6].

The dislocation shape for the row shaped cantilever category humidity sensor was obtained by static analysis as shown in Fig.

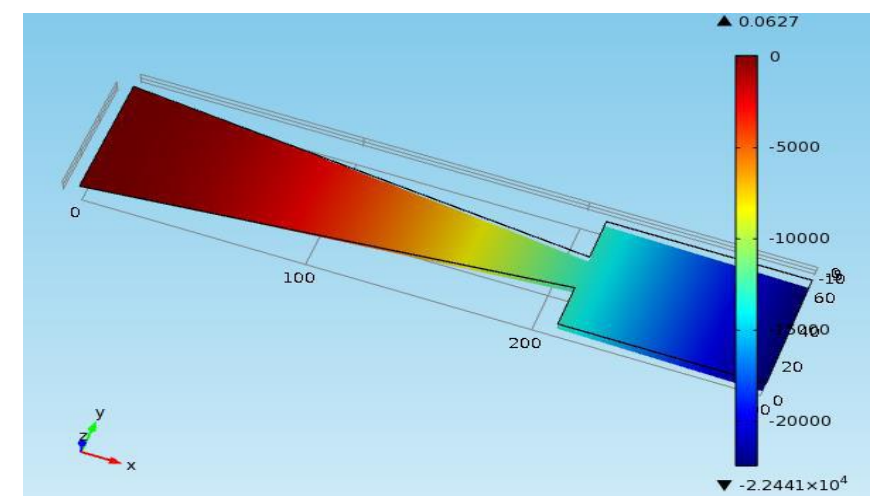

Fig. 2.Simulated result of paddle shaped type humidity sensor

The various structures has been designed and analysed and the values of displacements for rectangular, $\mathrm{V}$ shape and Paddle shapes are given in Table 1.

Table 1. Displacement analysis for various structures

\begin{tabular}{|c|c|}
\hline Structure & Displacement $(\boldsymbol{\mu m})$ \\
\hline Rectangular Shape & 3,000 \\
\hline V Shape & 7,000 \\
\hline Paddle Shape & 20,000 \\
\hline
\end{tabular}

\section{EXPERIMENTAL SETUP}

\section{A. Materials}

- Graphite Powder

- Hydrogen per oxide

- Concentrated sulphuric acid

- Deionized water

- Pottasium permanganate

\section{B. Material Synthesis}


Graphite powder was mixed with dilute hydrogen per oxide and concentrated sulphuric acid. The mixture was continuously stirred. After $8 \mathrm{hrs}$, the mixture was added with deionised water. The mixture is vacuum dried. Again deionised water is mixed and added with dilute hydrogen per oxide. The mixed is washed carefully and dried under vacuum. The hydrazine hydrate is added to the resultant solid graphene oxide which is dispersed in deionised water. After three days of room temperature, a black graphene oxide powder is obtained.

\section{Characterization}

The designed sensor can be imaged using Scanning Electron Microscope. To analyse the different materials present in the Graphene Oxide layer, X-ray diffraction process was also carried out.

\section{Measurement of Humidity in Graphene Oxide \\ Sample Using Gravimetric Method}

The humidity present in the graphene oxide can be expressed in terms of weight as the ratio of weight of water present in the dry to the dry weight of the graphene oxide sample. The graphene oxide weight was measured by gravimetric method which is shown in fig. 3

The moisture content is captivated by the graphene oxide is measured by drying the graphene oxide to constant weight and measuring the graphene oxide sample mass after and before drying. The amount of moisture content is the difference between the weights of the wet and dry samples. The drying of the graphene oxide is done by heating the sample in an oven at around $100^{\circ} \mathrm{C}$. This temperature is the boiling point of water content present in the sample and so it does not change any physical or chemical characteristic of the sample.

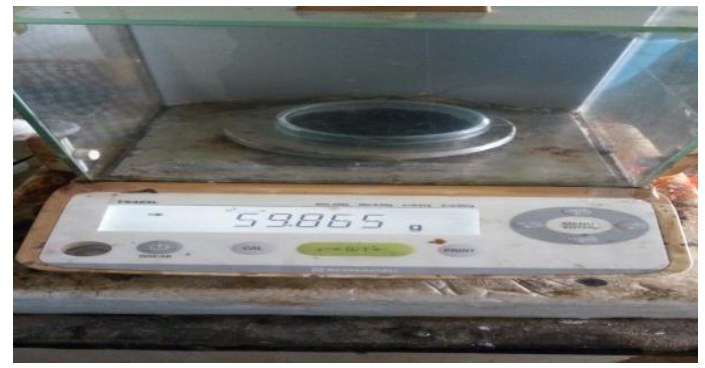

Fig. 3.Humidity measurement using gravimetric method

\section{Procedure}

- Weigh the glass pan

- $10 \mathrm{gm}$ of graphene oxide sample is taken in the pan.

- The weight of the sample after absorption of moisture is measured the recorded weight is for the wet sample (after absorption) and glass pan.

- The ample is heated in the oven for a temperature of around $100^{\circ} \mathrm{Cand}$ dried.

- Now the dry sample is weighted along with the glass pan.

- Now we can calculate the moisture content with the formula.

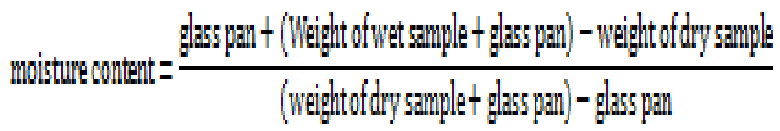

\section{RESULTS AND DISCUSSIONS}

In this study, the relative humidity is measured for various values of temperature. The relative humidity is measured by gravimetric object for different values of temperature and the plot is drawn between the temperature and Relative humidity as shown in Fig.4.

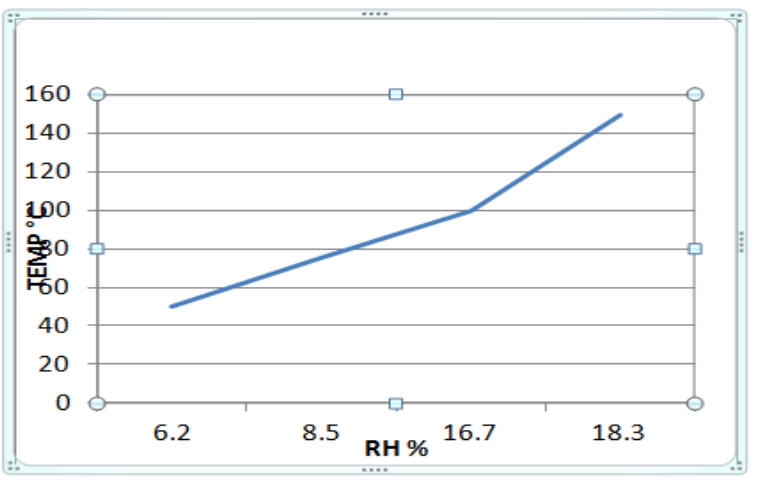

Fig. 4. Relative humidity variation for different values of temperature

A plot is drawn between different days having different exposure to moisture and the corresponding relative humidity is calculated. The plot drawn for days and relative humidity is shown in Fig.5.

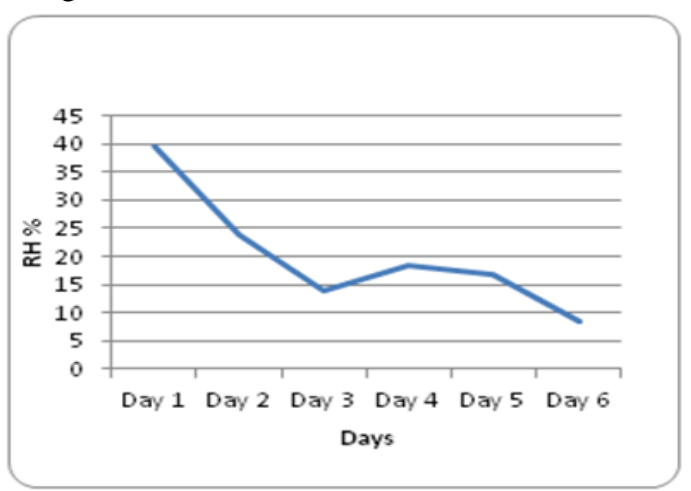

Fig. 5.Relative humidity variation for different days

\section{FABRICATION OF THE SENSOR}

Graphene oxide based cantilever type humidity sensor was fabricated .First the structure of displacement type of sensor is arranged. Then various structures like as rectangular type, V shape and paddle type were analyzed and from the simulation it is proven that paddle shaped MEMS humidity sensor has good sensitivity. The next step was the production of sensor by graphene oxide as the sensing material over by paddle shaped structure. The property of humidity was examined by doing characterization. Fig 6 shows the magnified image was characterized used through Scanning Electron Microscopy. (SEM).The Result for X ray Diffraction is shown as in Fig 6 . 


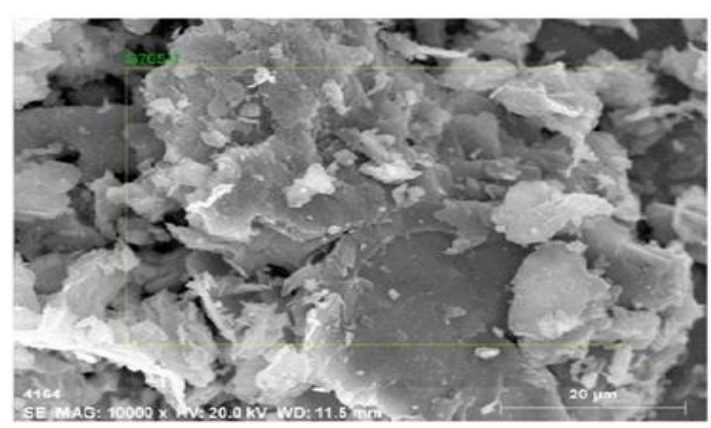

Fig. 6.SEM image of prepared Graphene Oxide.

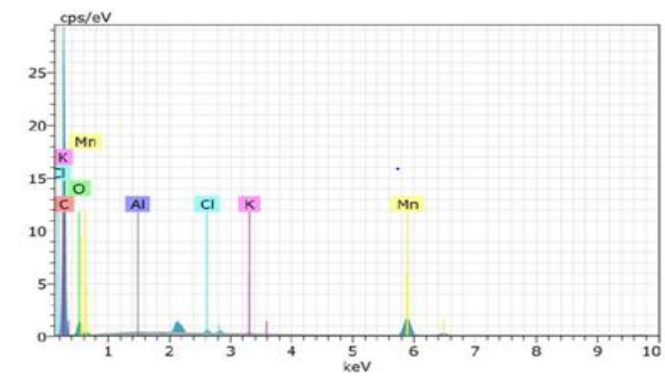

Fig. 7.X ray Diffraction of prepared Graphene Oxide

\section{CONCLUSION}

The proposed MEMS humidity device in this paper will be an effective sensor for monitoring the humidity of the oil in gear box lubricating system. Graphene oxide nano materials were successfully characterized and synthesized. The nano material obtained after synthesis was heated at various temperatures.XRD and SEM confirmed the formation of Graphene oxide nano material over the paddle shaped humidity sensor structures. The average particle size was greater and the porosity of the coated material increases with increasing temperature. The work done is a multidisciplinary engineering .The progress of this device arranges a significant fast response in measuring the humidity of lubricant oil condition monitoring and also the cost is reduced.

\section{REFERENCES}

1. Ivan Puchades,Lyanf.Fuller, "A thermally actuated microelectromechanical (MEMS) device for measuring viscosity," Journal of Microelectromechanical Systems, 20(3),June.2011 pp.601-608.

2. Daniel Arecco, "Analysis and preliminary characterization of a MEMS cantilever-type chemical sensor," InMechanical Engineering Department Worcester Polytechnic Institute, Dec.2003.

3. I. Dufour, E. Lemaire, B. Caillard, "D7. 4-MEMS-based Measurement of Rheological Fluid Properties," Proceedings SENSOR, May.2013, pp.598-603.

4. P. N. Prabhakaran, M. Renuga, "Design And Analysis Of Capacitive MEMS Viscometric Sensor For CGM", International Journal Of Emerging Technology And Advanced Engineering,2(11), Nov 2012,pp. 135 .

5. John K. Duchowski, H. Mannebach ,“ A novel approach to predictive maintenance: A portable, multi-component MEMS sensor for on-line monitoring of fluid condition in hydraulic and lubricating systems," Tribology Transactions, 49(4), Dec.2006, pp.545-553, 2006.

6. L. Chitra,Dr. V. Ramakrishnan, "A Novel design of capacitive MEMS pressure sensor for lubricating system,", IEEE National Conference on Emerging Trends In New \& Renewable Energy Sources And Energy Management (NCET NRES EM),Dec.2014, pp. 204-208.
7. A. A. Agoston, C. O“Tsch, B. Jakoby, "Viscosity sensors for engine oil condition monitoring-Application and interpretation of results," Sensors and Actuators a: Physical, 121(2), Jun.2005, pp.327-332.

8. J. DeBaecke, "Understanding and maintaining an effective lubrication system," Hydrocarbon Processing, 2009, 88(8), pp.27-27.

9. L.Chitra, Dr. V.Ramakrishnan, , "Design optimization of piezoresistive Multi MEMS device for lubricating system," Advances in Natural and Applied Sciences , 8(21),Dec.2014, pp.76-84.

10. L. Chitra,Dr. V. Ramakrishnan, "Comparative analysis of cantilever device for MEMS device for humidity measurement," Elysium journal of engineering research and management, Agu.2014.

11. N. Sujata, Naduvinamani, Mangesh R. Shanbhag, M. Gangotri, Bhat and Pooja Kulkarni, "Design and simulation of MEMS humidity sensor," World Journal of Science and Technology, 2(10), 2012, 23-26

12. A. R. K. Ralston, C. F. Klein, P. E. Thoma and D. D. Denton, "A model for the relative environmental stability of a series of polyimide capacitance humidity sensors," Sensors and Actuators B: Chemical, 34(1-3), Agu.1996, pp. 343-348.

13. Vijay K Varadhan K. J. Vinoy ,K.A. Jose..And MEMS, R. Their Applications. Pennsylvania State University, USA, Apr. 2003.

14. Z. M. Rittersma, "Recent achievements in miniaturised humidity sensors-a review of transduction techniques," Sensors and Actuators A: Physical, 96(2-3), Feb.2002, pp.196-210.

15. P.J.French , A.G.R .Evans," Piezoresistance in Pokysilicon and its application to strain Guages,"Solid state Electronics, 32(1), Jan.1989, pp. $1-10$

16. S. M. Firdaus, I. A. Azid, O. Sidek, K. Ibrahim, M. Hussien, "Enhancing the sensitivity of a mass-based piezoresistive micro-electro-mechanical systems cantilever sensor,"Micro \& Nano Letters, 5(2), Apr.2010, pp.85-90.

17. C. Pramanik, T. Islam, H. Saha, J. Bhattacharya, S. Banerjee, S. Dey “ Design, fabrication, testing and simulation of porous silicon based smart MEMS pressure sensor VLSI Design," 18th International Conference, 2005.

18. Duk-Soo Eun Dae-Young Kong Hyun-Jun Yoo Young-Myong Hong Jong-Min Jang Tae-Wook Kang In-Sik Yu Jong-Hyun Lee "Design and fabrication of a MEMS-based multi-sensor," In International Conference on Nano/Micro Engineered and Molecular Systems, Jan. 2008 pp. 588-591.

19. P. Maniraman, L. Chitra, "Comparative Analysis of Capacitive MEMS Pressure Sensor for Altitude Sensing," In National Conference on Emerging Trends In New \& Renewable Energy Sources And Energy Management (NCET NRES EM), Dec 2014, pp. 195-199.

20. A. Preethi, L. Chitra, "Comparative analysis of materials for the design of a highly sensitive capacitive type of MEMS pressure sensor," In National Conference on Emerging Trends In New \& Renewable Energy Sources And Energy Management (NCET NRES EM),Dec.2014, pp.1-8.

\section{AUTHORS PROFILE}

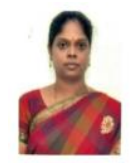

Dr. L. Chitra is an Associate Professor and Head in the department of EEE at Aarupadai Veedu Institute of Technology. She received her Bachelor Degree in Electronics and Instrumentation Engineering. She received her Master's Degree in Power Electronics and Drives in Anna University. She completed her PhD in Sathyabama Institute of Science and Technology in the field of Micro Electro Mechanical Systems for sensor design. Her research area includes MEMS, Renewable Energy, Process control, Sensors and Smart Sensors. She has published more than fifteen papers in International Conferences and Journals. She has a teaching experience of more than 18 years. She has received MNRE fund of Rs.1,50,000 for National Conference on Emerging Trends in New Renewable Energy Sources in the year 2014. She has also received a fund of Rs.1,00,000 from Unnat Bharat Abhiyan for Mobile Reverse osmosis based water purification system using Solar PV source for rural India in year 2019. She has also received a fund of Rs.50,000 from Unnat Bharat Abhiyan for DC Micro Grid based Solar PV generation to supply power to street lamp and common utilities in a village. 